\title{
Miura-ori, Basics for Designing its Folding Machines
}

\author{
Koryo Miura $^{\mathrm{a},}$ * \\ ${ }^{a}$ Institute of Space and Astronautical Science, JAXA \\ * Corresponding author
}

Abstract: The unique property of the Miura-ori map is due to the geometric property of "the single degree of freedom". With this, one can open a map with a single pull motion. However, due to this property, the high-speed folding machine is difficult to realized. In this presentation, author investigates the natural geometric properties of Miura-ori in detail and proposes a basic concept for designing its folding machine. Though, the result does not provide a draft of a folding machine, the basics for the design parameters is beneficial for future works.

Keywords: Miura-ori, foldable map, geometry, folding machine

\section{Introduction}

A novel form of folded map, presented first at the ICC1980Tokyo, was gradually accepted by the cartographic community. Now, the transit rout map of Tokyo city in the form of so-called Miura-ori became a standard in this category.

The original concept of this deployable structure was due to the author's study on deployable solar array for "Solar Power Station” outside the Earth (Miura et al. 1985). The foldable map was used for a model to convey this concept of deployable structure at the International Astronautical Federation, Tokyo 1980 (Miura, et al. 1980).

\section{Map of Venezia, presented at ICC1980Tokyo}

In 1978, Olivetti Corporation of Japan provided me a generous offer to publish my idea for the article and the model in SPAZIO, the company's celebrated magazine.

Figure 1 shows the folded state, and Fig. 2 shows the central part of the map in the deployed state. The content of this map is provided by Prof. Jinnai, as an article "The Past and the Present of Saint Margherita's Square in Venice”, in the same issue.

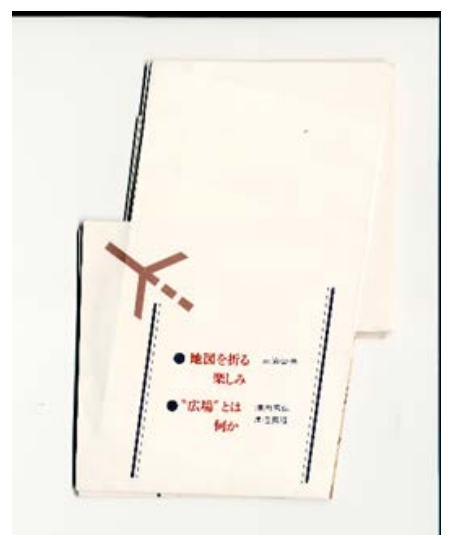

Figure 1 Map of Venezia (folded state)

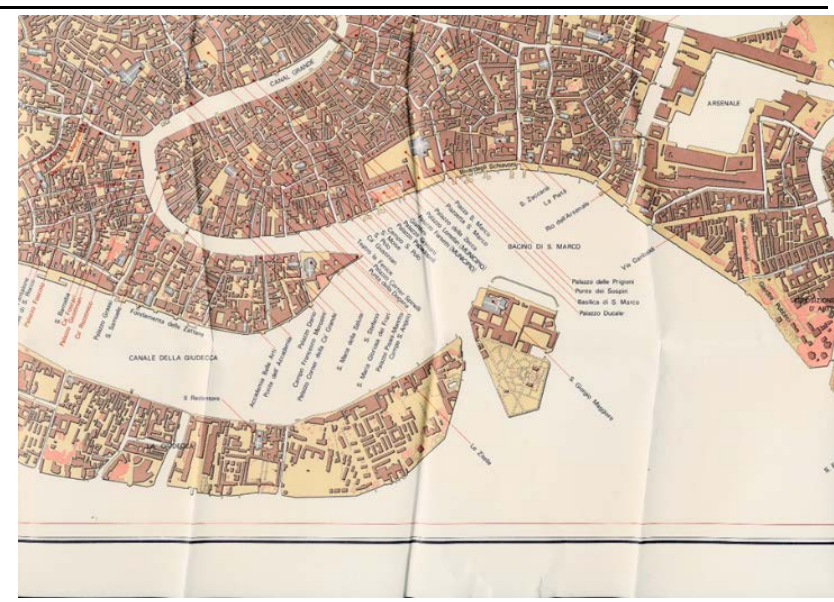

Figure 2. Map of Venezia (deployed, local)

The map was distributed to the participants at the conference room. The zigzag fold lines can be seen in Fig. 2. The fold lines are about 6 degrees inclined from the vertical direction. After 40 years, I noticed, there is no sign of rent everywhere. The reason is that, due to the single degree of freedom system of this design, there is no chance to reverse any fold. Because, every mountain and valley folds are memorized in it, there is no chance to reverse them. Exactly, there is only one case to reverse, if all folds are simultaneously reversed.

\section{Thin plate subjected to bi-axial displacement}

.Miura put forward the hypothesis that this periodic surface is the extreme outcome of a vanishingly thin, elastic, flat plate subjected to bi-axial, uniform displacement. Figure 3 depicts the subject, where a thin square sheet is displaced uniformly from its borders simultaneously (Miura, 1970). 


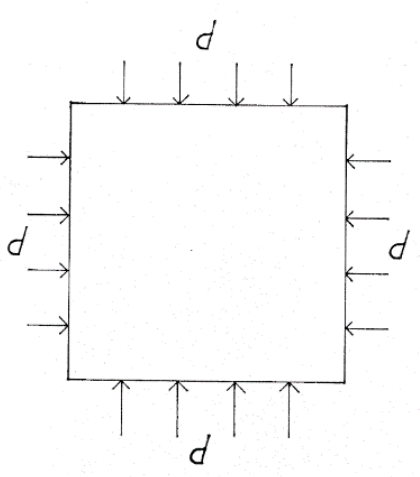

Figure 3 Subject of bi-axially displaced thin plate

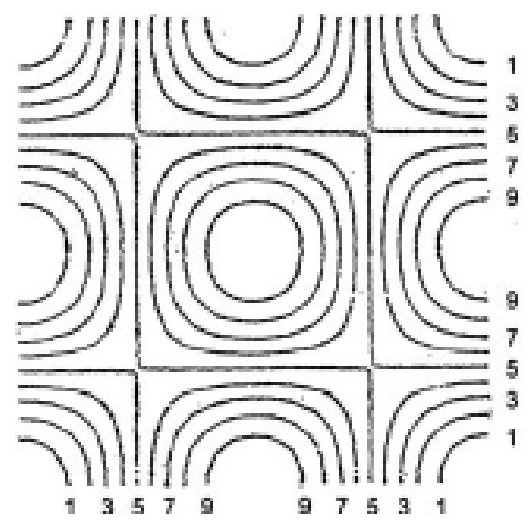

Figure 4a Displacement pattern (thickness level is normal)

Figure $4 \mathrm{a}$ is the initial buckling mode, assumed to be periodic. The numbers of the contour lines denote the largest downward (counter 1) and upward (contour 9) deflection. In other word, this is the typical geographic map to express the array of independent hills and ponds distributed periodically.

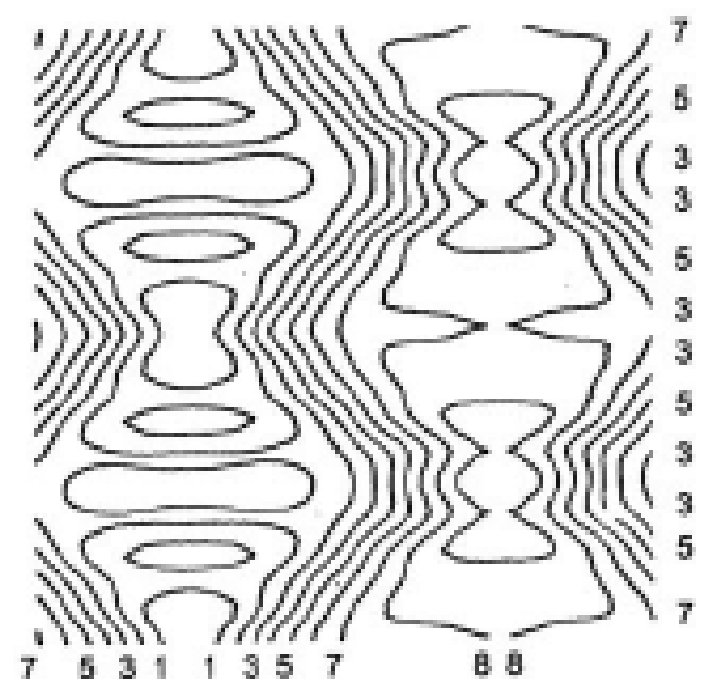

Figure 4b Displacement pattern (thickness level is medium)
As the relative thickness of the plate is reduced. the hills and ponds are integrated to form the mountain-and-valley (zigzag) pattern (Fig. 4c).

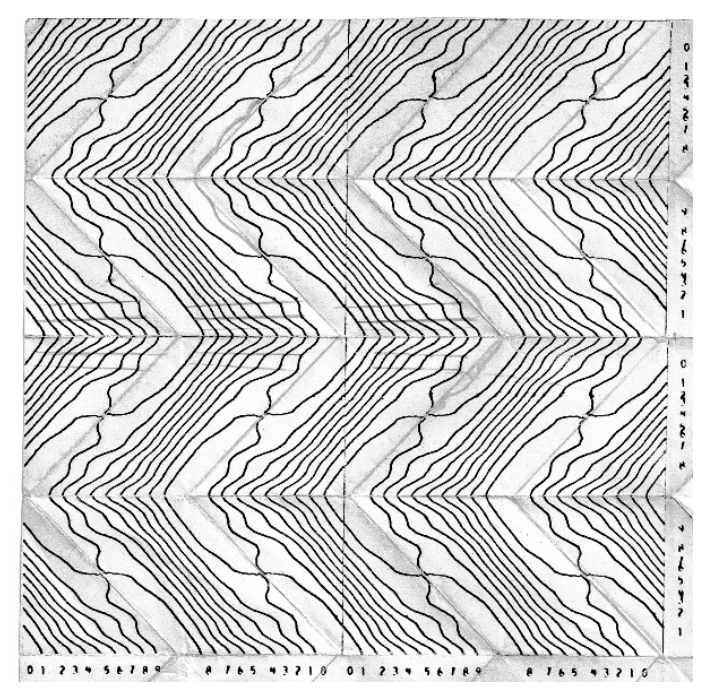

Figure 4c Displacement pattern (thickness level is low).

Fig. 5 shows an ultimate low strain energy, fully developable displacement pattern that matches the repeating unit of Miuraori.

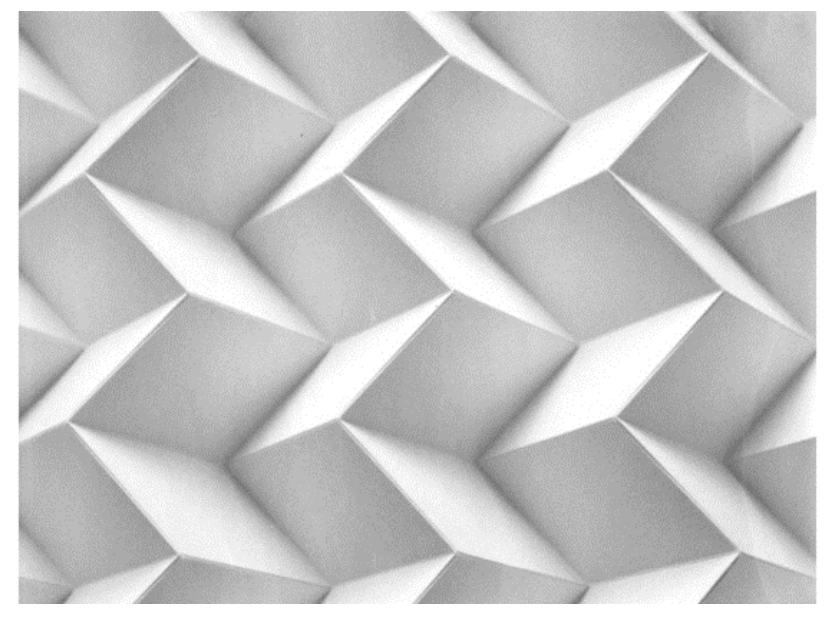

Fig.5 Ultimate displacement pattern (model) 


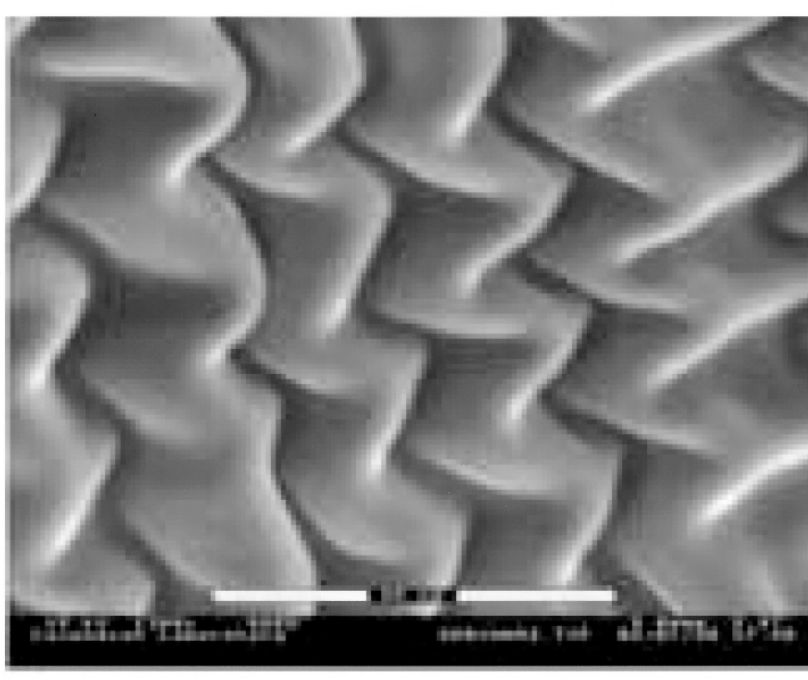

Figure 6 Experimental proof of Miura-ori (Mahadevan, and Rica)

In 2005, Mahadevan and Rica of Harvard University found the experimental proof of Miura-ori. The bi-axial compression of a thin, stiff, elastic film, supported on a thick soft substance, yields into a Miura-ori.

These studies, a computational experiment and a physical experiment, predict a possibility of "bi-axial folding” of a thin sheet of paper theoretically. In the following section, the essential geometry of Miura-ori is briefly presented that is vital to design of the folding machine.

\section{Mathematical foundation on bi-axial folding}

In deployable structures, one distinguishes between two different types of deployable behaviours; the synchronous deployment for a structure that deploys as a whole, and the sequential deployment for a structure that deploys one piece at a time. deploys synchronously. In fact, this synchronous deployment behaviour of Miura-ori is the source of difficulty for designing the folding machine.

Miura-ori is based on a repeating unit cell consisting of four identical parallelograms with side length $a$ and $b$ and with internal angles $\alpha$ and 180- $\alpha$. The four parallelograms are shown in Fig. 7a for two different configurations of Miura-ori. The geometry of the partially folded unit cell is shown in Fig. 7b. The overall dimensions of the repeating unit are defined by its height, $\mathrm{H}$, measured in the $\mathrm{z}$-direction, and the $\mathrm{x}$ and $\mathrm{y}$ distances, respectively $2 \mathrm{~S}$ and $2 \mathrm{~L}$ between the corners of the unit, lying in the $\mathrm{x}-\mathrm{y}$ plane. (a)

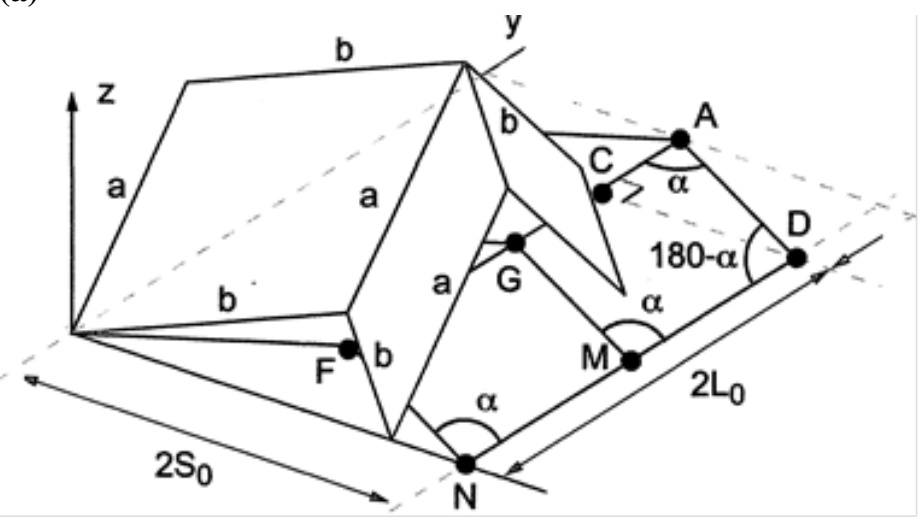

(b)

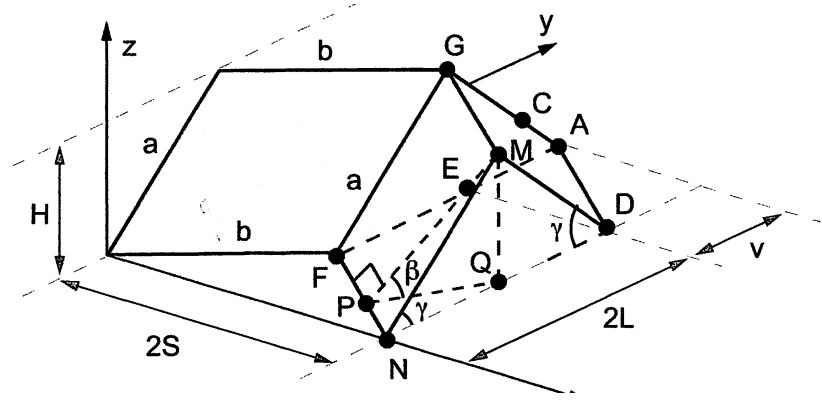

(c)

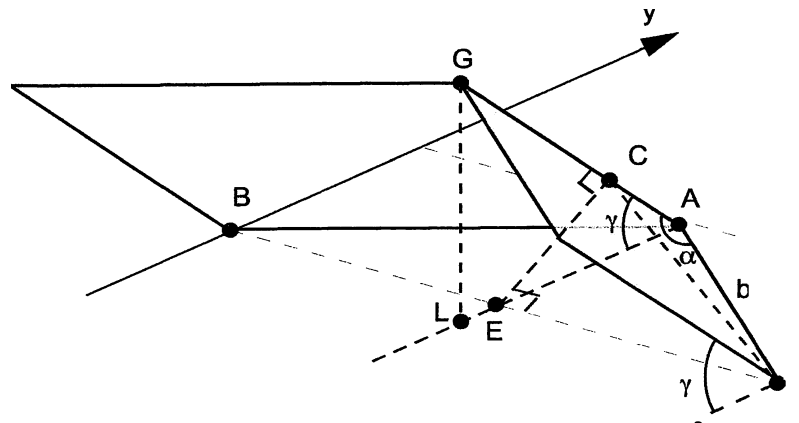

Figure 7 Geometry of Miura-ori unit

A further dimension $\mu$ is defined in Fig.7b. it identifies to the edge nodes. In Fig. $7 \mathrm{~b}$ the dihedral angle $\beta$ between any of the four parallelograms and the $x-y$ plane is defined as the deployment angle of the unit cell. Hence $\beta$ $=0^{\circ}$ when the unit cell is fully deployed i.e. it is flat, and $\beta=90^{\circ}$ when the unit is fully folded, the four parallelograms overlap. An alternative deployment angle, $\gamma$, is also defined in Fig. 7b. $\gamma$ is the angle between the creases lines that are straight in the flat configuration, e.g. $\mathrm{DM}$ and $\mathrm{MN}$, and a line parallel to the y-axis. Fig. 7c shows a detailed view of only two parallelograms in the unit cell.

A simple relationship between the angles $\alpha, \beta$ and $\gamma$, is expressed as follows:

$\sin \gamma=\sin \alpha \sin \beta$ 
Since $\alpha$ is defined for any chosen Miura-ori, for any value of the deployment angle $\beta$, the alternative deployment angle $\gamma$ can be determined from the above equation.

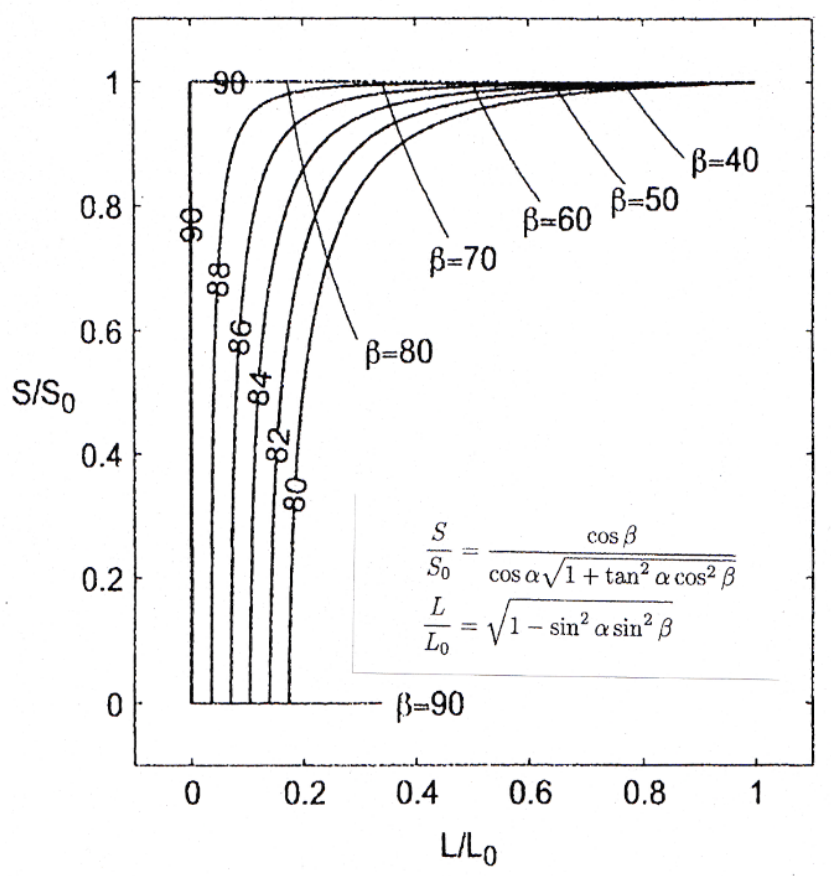

Fig.8 Biaxial expansion of Miura-ori unit cell with different angles

The ratio between the dimensions of the unit cell in a general configuration and the flat configuration, $\mathrm{S} / \mathrm{S}_{0}$ and $\mathrm{L} / \mathrm{L}_{0}$ are shown in Fig. 8. Plot of these expression for deployment angles between 90 degree and 0 degree can be used to study the deployment coupling of Miura-ori. Several values of $\alpha$ has been considered in Fug. 8. The general trend is for the packaged unit cell to initially expand mostly in the $\mathrm{x}$-direction and then mostly in the $\mathrm{y}$ direction. An extreme behaviour (decoupling) is achieved for $\alpha=90$ degrees, as Miura-ori approaches the map folding. For $\alpha=90$ degrees, the plot of $S / S_{0}$ vs $L / L_{0}$ no longer consists of a smooth curve, but instead it breaks into two perpendicular-straight lines.

\section{Strategy for designing folding machines}

The most difficult problem for designing the Miura-ori folding machine is the coupling of the folding, in S and $\mathrm{L}$ directions. The coupling is also the most important behaviour of the Miura-ori. The first Miura-ori map of Venezia shown before has the angle $\alpha=84$ degree. It is the limiting case, where the step-by-step multi-moulds could be used for production.

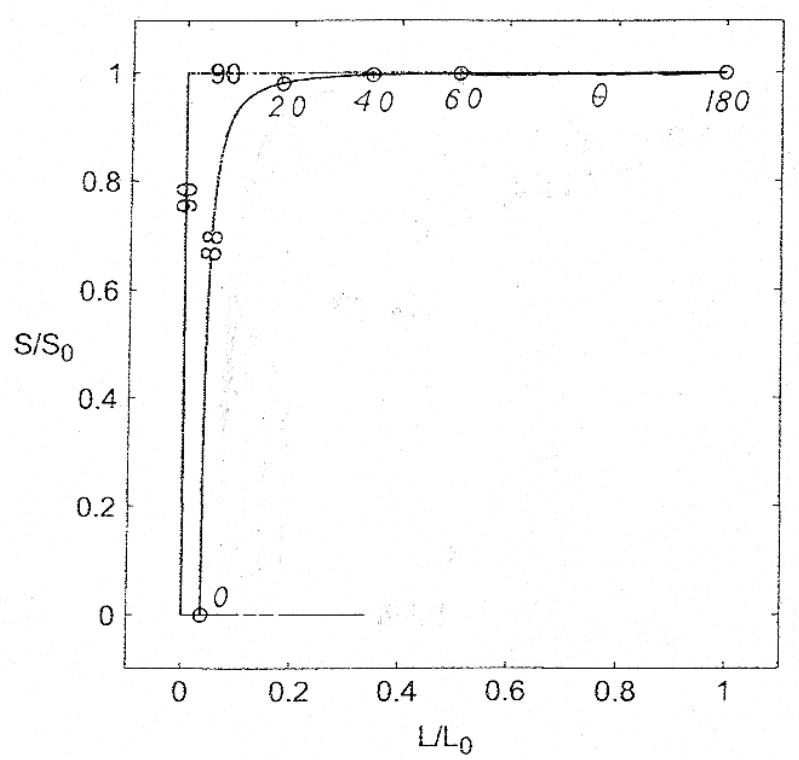

Fig. 9 Scenario for folding procedure

The present author proposes a "follow the Law of Nature" procedure for solving the subject. The formation of Miura-ori, discussed in the previous sections, reveals the nature of folding process under external compression. If we can simulate the process carefully, we should be able to establish a Miura-ori folding machine.

A possible scenario to solve the problem is use of the curve, closest to the $\alpha=90$, for instance, $\alpha=88$. The curve is shown in Fig. 9, where the curve and the relevant formed ridge angle, $\theta=180-2 \beta$, started from 180 degree (flat) to 0 degree (sharp).

The first stage starts from $\theta=180$ degree (flat) to around 40 degree. During this stage, the machine simply compresses the sheet laterally (L-direction) to form hills and valleys shape.

At the second stage, a small amount of vertical compression (S-direction) is applied to form a slight zigzag pattern to the hill and valley ridges. This is the initiation of Miura-ori pattern, and some tools to fix the ridges can be used at this stage.

The third stage could not be done with the machine setting dedicated to the L-direction process.

Therefore, the partially folded piece should be removed from the machine, and it will be folded by a separate machine dedicated to fold the piece in the S-direction to obtain the final result.

\section{Concluding remark}

The author provides a basic geometric strategy for folding machine dedicated to Miura-ori. The author believes that the real folding machine might be designed with the similar principle. 


\section{References}

Mahadevan, L., Rica, S. (2005). Self-organized origami, Science, Vol. 37, 1740

Miura, K. (1970) Proposition of pseudo-cylindrical concave polyhedral shells, Proc. IASS Symposium, September-October, Vienna

Miura, K. (1978). The fun of map folding, SPAZIO, Olivetti Corporation of Japan, Vol. 19, 72-83 (in Japanese and English)

Miura, K., Sakamaki, M. (1980). A novel design of folded map, 10th International Conference of International Cartographic Association, Tokyo

Miura, K. (1980). Method of packaging and deployment of large membranes in space, 31st Congress of International Astronautical Federation, Tokyo

Tanizawa, K., Miura, K. (1978). Large Displacement Configuration of Bi-Axially Compressed Infinite Plate,

Trans. Japan Society for Aeronautical and Space Science, Vol. 20, No. 50, pp.177-187 